\title{
ZUR ETYMOLOGIE VON BRAUT.
}

Von Braune in diesen Beitr. 32, $30 \mathrm{ff}$. semantisch beleuchtetes germ. $b r \bar{u} p s, b r \bar{u} d$ etc. ${ }^{1}$ ) ist zweifelsohne zum ital. beinamen der Venus, Frütis oder Frutis, zu stellen (s. Beitr. 32, $58 \mathrm{f}$. und 34,562; wegen nicht zu fixierender quantität des $u$ vgl. von Kluge an letzter stelle bemerktes). Dagegen ist in Corssens Aussprache 2², 206 mit Frŭtis verknüpftes lat. frutex fernzuhalten, folglich dieses wort auch nicht zu brüps etc. zu stellen: 'strauch, staude' lässt sich schwerlich weder mit der von Braune dem germ. subst. zuerkannten grundbedeutung 'beischläferin' noch mit der von Kluge (Beitr. 34, 565) für das nomen als denkbar erachteten semantischen basis 'brotkneterin' noch auch mit einer grundbedeutung 'die sich vermählende' (s. unten) vereinbaren. Ich möchte aksl. bra-kī 'nuptiae' beiziehen, dessen auf $\bar{o}$ zurückzuführendes $a$ auf altem langdiphthong $\bar{o} u$ beruhen kann, zu dem der stammvocal von Fr $\breve{u} t i s$ und brūps etc. im ablaut stand. So hätten das ital. und das germ. wort zu gelten als -ti-derivatum mit ursprüng-

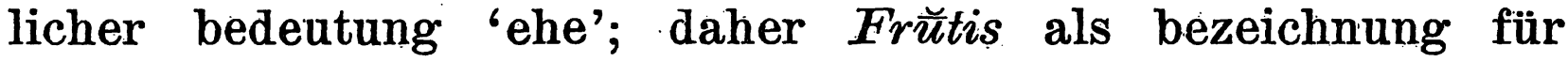
'göttin der ehe' (vgl. Veiuus 'göttin der wonne' = aid. vanas 'wonne') und vorgerm. bhrūtis = 'diejenige, an der die ver-

1) Den gedachten erörterungen gemäss sind auch für in den afries. quellen begegnendes breid die bedeutungen 'braut am hochyeitstage' und 'neuvermählte' geltend zu machen:

als hy sȳn(e) breid halla bez. halet, und hwerso een man sȳn breyd haleth (belege in v. R.'s Wb.) - sa fulgat thio breyde tha lȳke (des im brautzuge erschlagenen bräutigams) (bel. Zur altostfrios. lexic. 27);

thiu breid ... birāwat ... hive stiūrcgonges (des kirchganges), und hwersa ma ène breid biräwat ande hire kerekgonge (belege in $\nabla . \mathrm{R}$.'s Wb.), sowie Thiu breid ... birāwat hire fiuurgonges iefta stiūrcgonges $\nabla . \mathbf{R}$. $\mathbf{E}^{1}$ 232, 1 ff.; vgl. hiermit Zur altostfries. lexic. 38 aus $F$ citiertes: and hio breydelike (als neuvermählte) sinne (des ehemannes) bethselma (bettstelle) urstop (beschritt) and on tha bedde hire tinues nette (genoss) mitha monne and a morna (am morgen darauf) upstōd, to tzū $r k a$ geng etc.; in bezug auf hire fiuurgonges gilt breid für 'braut am hochzeitstage' (vgl. obigem citat aus $F$ vorangehendes Thet thio frīe Fresinne kome on thes frèia F'rēsa were (besitz) .... mith bèkana brande, d.h. 'beim leuchten der hochzeitsfeuer'). 
ehelichung zu tage tritt', d.h. 'die sich vermählende, die braut am hochzeitstage'. Aus solcher bedeutung aber lassen sich alle die von $\mathrm{Br}$. für brüps etc. hervorgehobenen anstandslos herleiten:

einerseits durch übertragung von antecedens auf das sequens 'neuvermählte'1), woraus durch beziehung der jungen ehefrau auf die eltern des sohnes 'schwiegertochter' bez. durch begriffserweiterung 'gattin' (aus 'gattin' auch gelegentlich durch begriffserweiterung 'weib', vgl. Beitr. 32, 52);

andererseits durch begriffserweiterung 'die sich über kurz oder lang vermählende', also 'verlobte', woraus ebenfalls durch erweiterung 'heiratsfähige jungfrau'; [man beachte in Beitr. 32, 49 angeführtes mengl. brud, bruid etc. = 'junges weib, mädchen' sowie in Zs. f. d. wortf. 1, 246 nach Delbrück hervorgehobenes neuslav. nevjesta $=$ 'verlobte' und 'erwachsenes mädchen' und vgl. auch ndl. vrijster = 'verlobtes mädchen' und 'heiratsfähiges mädchen' (in beiden bedeutungen nur verwant für mädchen unteren standes) und 'unverheiratetes frauenzimmer' (in dem ausdruck oude vrijster 'alte jungfer')].

Wegen entstehung einer gelegentlichen mnl. mhd. bedeutung 'illegitime beischläferin' und wegen verwendung von mhd. briuten, mnd. mnl. bruden ( $u$ als schreibung für $\ddot{\ddot{u}}$ ) 'beschlafen' vgl. Beitr. 34, $562 \mathrm{f}$, Eine parallele zur oben erörterten begrifflichen entwicklung bieten zu krimgot. marzus 'nuptiae zu stellendes lit. martì 'braut am hochzeitstage', 'junge ehefrau', 'schwiegertochter', kret. $\mu \alpha$ ó $\tau \iota s$ 'jungfrau' (= 'die heiratsfähige').

Eine wesentliche stütze erhält die vorgeschlagene deutung durch in Beitr. 32, 41, fussn. aus den Ahd. gll. citierte, zu nurus, bruta stehende proatun, -on Ra und $\mathrm{K}$, brǒt $\mathrm{Clm}$. 13002, wofür Braune, offenbar als notbehelf, die vermutung aufstellt, dass hier eigentlich prut als übersetzung von nuris, bruta gemeint war bez. brŏt als schreibfehler für brut zu gelten hätte. Kluge erhebt Beitr. 34, 565 die frage, ob in proatun, -on nicht oa als

1) Als genaue entsprechungen begegnen $\nu v \dot{\mu} \varphi \eta, \nu v \mu \varphi l o s=$ 'braut, bräutigam am hochzeitstage' und 'junge ehefrau, junger ehemann'.

Eine ähnliche metapher beobachtet man in ahd. gemahelo, $-a$, mhd. gemahel( $e$ ) 'verlobte(r)' und 'verheiratete(r)', mlat. sponsus, -a 'verlobte(r)' und 'neuvermöhlte(r)'. 
schreibung für aus altem au contrahiertes $\bar{o}^{\alpha}$ vorliegen dürfte und das wort demnach auf brōt zu beziehen wäre, so dass die parallele desselben mit ags. hlófdize in ihr recht träte und ein bei fassung von proatun, -on als obliquem casus anzusetzendes schwaches femininum brōta sowie damit zu verbindendes brüt als koseformen zu einem volltypus wie hlófdize zu verstehen wären. Nun könnte man allerdings das oa der Raund der K-glosse als die nach Kögel (Zum Ker. glossar 23. 24) neben normalem $o$-zeichen mitunter für $\bar{o}^{a}$ verwante schreibung gelten lassen. Was aber dânn mit brǒt in Clm. 13002 anzufangen, das nur bruot repräsentieren kann, indem brout (die brǒt überliefernde hs. hat bekan̄ntlich ó zur darstellung sowol von $o u$ als von $u o$ ) aus lautgesetzlichem grund aúszüschliessen? Ausserdem aber ist nicht zu übersehen: erstens dass gemäss Kögels bemerkung (Zum Ker. gloss. $151 \mathrm{f}$.) übersetzung eines nom. nurus, bruta durch acc. proaton, -un nicht glaubhaft erscheinen kann; zweitens dass wir es der überlieferung zufolge in proaton, -un und brǒt mit ausdrücken zu tun haben für 'schwiegertochter' (nicht für 'neuvermählte' oder 'ehefrau'). Alle schwierigkeiten schwinden indessen, wenn man in oa der normalen verwendung dieses zeichens gemäss eine schreibung erblickt für aus altem $\bar{o}$ entstandenen diphthong und, dem von Kluge (Beitr. 34, 565) gegebenen fingerzeig folgend, -un, -on (vgl. Kögel s. 151) auf alte femin. endung -uni (aưs -uñ̄) zurückführt, dem diphthong der beiden nominalbildungen zu grunde liegendes $\bar{o}$ aber mit dem $a$ von aksl. $b r a-k \bar{\imath}$ in eine linie stellt, d. h. ebenfalls auf altes ôu zurückführt. So erhalten wir ein mit -ti- gebildetes, altes verbalabstractum bhrö(u)ti-, das durch übertragung der alten bedeutung 'ehe' zur bezeichnung 'einer durch die ehe (des sohnes) erworbenen tochter' verwant werden konnte; hieraus vorhd. brōd, das direct brǒt ergab ${ }^{1}$ ), indirect dem proatun, -on zu grunde liegt, das durch erweiterung nach art von zu brüt gebildetem mhd. brütinne entstand.

Als nebensächliches betreffend seien ferner an Braunes dankenswerte untersuchung noch folgende bemerkungen angeknüpft.

1) Ahd. gll. 4, 89, 21 bald nach brǒt begegnendes brǒtigoum braucht kein schreibfehler zu sein: es kann eine gelegentlich durch einwirkung von brǒt für brūtigoum eingetretene neubildung repräsentieren. 
Statt an ehti (d.h. an êhti) 'in ehelichem besitz' in Hēl. 507. 2707 (sīthor siu mannes warth, erlas an èthti - thiu err sines bruother was idis an è hti) bietet $\mathrm{M}$ ant(h)ehti, offenbar, wie Br. (Beitr. 32, 32, fussn. 1) bemerkt, als die folge des umstandes, dass dem schreiber von $M$ besagte wendung unbekannt war. 1) Dass indessen dieser copist in der nicht verstandenen wendung beide male gerade an $\mathrm{zu}$ ant- verderbt hätte, müsste auffallen. Vielmehr berechtigt ant- zur vermutung, dass wir es hier mit einer dem schreiber des $M$ geläufigen und von ihm für an ehti seiner vorlage eingesetzten form ant(h)ehti (d. h. antēhti) zu tun haben, d. h. mit einer neubildung, die, nachdem die alte, nicht mehr als präpositionelle verbindung empfundene wendung dem in den unflectierten casus auf $-i$ ausgehenden adjectivischen -io-stämmen gleichgestellt war, sich durch entlehnung des ant- von anthet $i$ 'verlobt' entwickelt hatte.

Die tatsache, dass . aus westgerm. dialekten herrührende franz. $b r u(t)$ und rhätoroman. brit, breit als benennungen für 'schwiegertochter'. gelten, während in den westgerm. quellen kein briud, brūt etc. = 'nurus' begegnet, möchte Br. (Beitr. $32,44)$ so erklären, dass das wort in seiner eigentlichen bedeutung 'neuvermählte' entlehnt sei, die entlehnungen aber, nachdem sie auch für 'schwiegertochter' in schwang gekommen waren, die eigentliche bedeutung gänzlich oder etwa, mit nur vereinzelter erhaltung derselben, aufgegeben hätten (in gewissen nordfranz. mundarten gilt bru nach Tappolet, Die roman. verwantschaftsnamen s. 131 auch für 'nouvelle mariée'; doch ist hieraus, im hinblick auf nurus 'schwiegertochter' und 'junge ehefrau', nicht notwendig auf franz. erhaltung der alten bedeutung zu schliessen). In gleicher weise wäre nach Br. aus dem ostg'erm. entlehntes brītis für 'schwiegertochter' in schwang

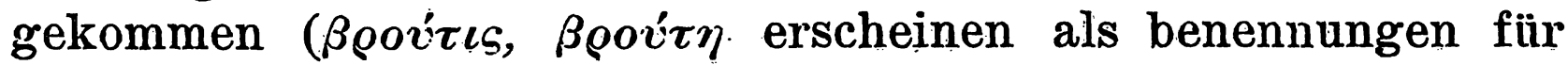
'junge frau', vgl. Zs.f.d. wortf. 1, 240 ff.); got. in Matth. 10, 35 belegtes $b r \bar{u} p$ s sei nur gelegentlich für 'nurus' verwant (Beitr. s. 38), denn aus brüpfaps gehe die eigentliche bedeutung des femin. personennamens hervor. Doch ist nicht einzusehen,

1) Wegen eines der as. wendung entsprechenden salfrk. an ehti $=$ 'in (durch verlobung' exwirktem) besitz', also 'verlobt' vgl. Beitr. 25, $329 \mathrm{f}$. Wegen des ndl. auf ëht 'ehe' ('ehelicher besitz') zurückgehenden echt s. Tijdschr. voor nederl. taalkunde 20,303. 
warum die geltung von got. brüps für 'nurus' nur eine occasionelle sein sollte und brütis 'schwiegertochter' der aus dem Unterdonauland herrührenden inschriften nicht auf directer entlehnung aus brüpiz 'nurus' beruhen könnte. Was aber die franz. und rhätoroman. lehnwörter betrifft, so möchte man fragen, ob sich nicht eher die annahme empfähle, dass in den gebenden (nfrk. und alem.) dialekten, wie im got., neben 'neuvermählte' auch die hieraus abgeleitete bedeutung galt und die entlehnenden Franco-Gallier bez. Rhätoromanen, dem bedürfnis nach einem ausdruck für 'des sohnes frau' genügend, ein in dem sinne verwantes fränk. bez. alēm. wort aufgenommen hätten; begreiflicher wäre eben solcher vorgang als der oben erwähnte, d. h. nicht zielbewusste entlehnung von brüd, brüt 'neuvermählte', die erst indirect zu verfügung über den erwünschten terminus führen konnte.

Wegen bruta der glossen vgl. Braune s. 42.

\section{GAB ES EINEN \\ GOT. NOMINATIVUS ABSOLUTUS?}

Die heikle frage, ob bez. inwiefern der got. sog. absolute dativ auf einen vom finalen verbum des satzes abhängenden dativ und ein diesen casus begleitendes partic. zurückgeht (vgl. Streitbergs Got. gr. $\$ 260$ und die daselbst angeführte literatur) oder als alte, mit aid. absol. locativ, gr. absol. genetiv, lat. absol. ablativ in eine linie zu stellende, eigentl. temporale construction zu gelten hat, lasse ich unerörtert. Mich auf feststehendes beschränkend, erinnere ich an die bekannte tatsache, dass der gr. gen. absol. von Wulfila nicht selten durch einen mit mi $\not p p a n e i, ~ b i \not p \bar{e}$ eingeleiteten hypotaktischen satz, einmal auch, in Matth. 27,57, durch einen satz mit pan über-

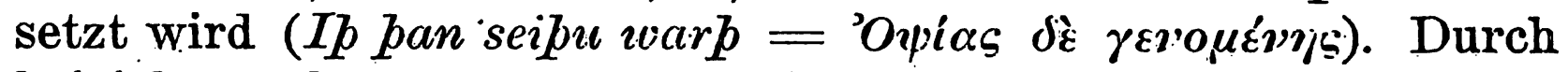
beiziehung letztgenannter version wäre $\mathrm{m}$. e. dem in Mark.

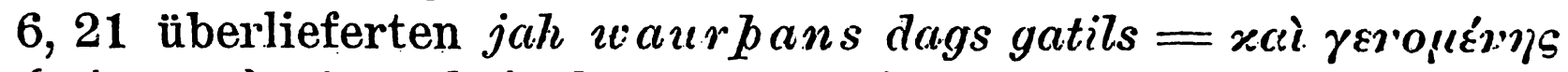

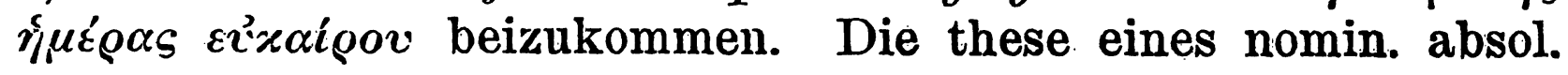

